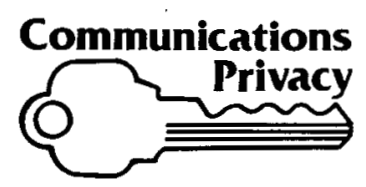

\title{
Security of Computer Communication
}

\author{
Dennis K. Branstad
}

\section{Cryptographic algorithms and systems can provide high levels of security when computers are connected in a distributed processing network.}

Security of digital computer communications is rising on the list of computer security requirements as the interconnection of computers becomes more commonplace. The reasons for interconnecting computers include improved service, improved reliability, and improved information. However, there are risks in connecting computers. A recent newspaper article reports a story about a woman who was told that she could not make a deposit in her bank account on a certain day from a branch bank. When she asked why, the answer was "because its raining too hard." The reply was absolutely accurate. When the intensity of the rain exceeded some threshold, the communications between the terminal in the branch bank and the computer in the central bank became garbled because of wet telephone connections and all interbank operations had to be suspended.

The security provided by the techniques described in this paper will do nothing to alleviate this problem. In fact, the techniques cannot solve many of the practical security problems encountered in computer operations. They will provide, however, high levels of security against certain types of real threats, both existing and

The author is with the Institute for Computer Sciences and Technology, National Bureau of Standards, Washington, DC. potential, that arise when terminals and computers are connected in a distributed processing network.

Cryptography is a method of secret writing. It is a way of hiding the content of a message through a positionscrambling process or through some other method of transformation. The original message is typically called plaintext and the transformed message is called ciphertext. The objective of cryptography is to scramble the message in such a way that an unauthorized and unintended recipient of the ciphertext cannot recover the underlying plaintext, while an authorized and intended recipient can easily do so. Cryptography cannot alleviate the communication problem caused by the rain because it is necessary that the ciphertext get from a transmitter to the receiver unperturbed. Cryptography cannot prevent intentional jamming of communications or accidental modification of communications. It will, however, help to detect them and prevent unwanted side effects of their occurrence. The prime benefits of cryptography are the prevention of theft and the intentional, unauthorized modification of messages in a communication system.

Transforming plaintext to ciphertext is called enciphering or encrypting. The method used for transforma- 
tion is known as an encryption algorithm. It expresses the set of rules for performing the transformation. For communications purposes an inverse transformation must be used to convert the ciphertext to plaintext. The operation is called deciphering or decryption. The decryption transformation need not be identical to encryption and may appear quite different, but still will be its inverse. In addition, the enciphering operation need not be the inverse of the deciphering operation, i.e., the encryption operation must be done first. Classical cryptographic systems, however, use enciphering and deciphering transformations which are the inverses of each other and are similar, if not identical.

Security objectives which can be met through the use of cryptography include: 1) prevent disclosure of plaintext, 2) prevent selective modification of plaintext, 3) prevent insertion of false plaintext, 4 \} detect modification of ciphertext. When used in conjunction with a message numbering system, cryptography can: 5) detect deletion of messages, 6) detect replay of messages. In

\section{Security in computer communications serves not only to prevent disclosure of the original message; other objectives include protection against alterations or introduction of false messages to the plaintext}

all cases, it is assumed that an intelligent opponent or antagonist has physical access to the communication line being protected, but does not have access to the terminals on either end of the line. Physical security must be provided to prevent access to the terminals.

\section{CRYPTOGRAPHIC ALGORITHMS}

Cryptographic algorithms are easy to develop. There exist many mathematical transformations which are inverses of each other. Addition and subtraction are inverse transformations; the Exclusive-Or (Binary addition without carryl. operation is its own inverse. The Exclusive-Or (XOR) operation is often used as a fundamental operation in a cryptographic algorithm. Random numbers, when XORed to data, make the results random. The same random number, if XORed to data twice, results in the original data. This fact is used in many cryptographic algorithms in which a pseudorandom number is generated and XORed to the data during encryption. The identical pseudorandom number is generated and XORed to the ciphertext during decryption. The security of the data depends entirely on the cryptographic characteristics of the random number generator.

Even though cryptographic algorithms are easy to develop, the level of security that they provide is difficult to measure. However, a good cryptographic algorithm has the following general characteristics.

1) The encryption and decryption operations should be simple and efficient for authorized users.

2) The decryption operation should be very difficult for unauthorized users.

3) The security of the data should not require that the algorithm itself be kept secret.
4) The efficiency and security of the algorithm should not be data dependent.

Cryptographic algorithms use a combination of functions operating on some data (plaintext) under the control of a parameter, known as a key, to transform the data into ciphertext. Binary algorithms operate on binary data of a fixed or maximum length. The result of the encryption operation is generally of the same length as the original data. If the result is shorter and data compression techniques have not been used, information has been lost and the original data cannot be recovered. Algorithms which discard information on purpose are called one-way algorithms and can be useful in certain applications, e.g., password encryption in which the original password can never be recovered from the ciphertext, but can be matched with the shorter ciphertext form for user authentication. If the result of the encryption operation is longer than the original data, more bits must be transmitted or stored, resulting in reduced effective communication speed or in increased data storage costs.

Cryptographic algorithms can be categorized in various ways. One method relates to how the key is used in the algorithm. Almost all algorithms use one or more parameters which are selected by a group of users to be used for some time period. The parameters chosen are called the cryptographic key and the time period is called the cryptographic period. The key should be chosen from all the possible keys in a random fashion and the choice should be unrelated to previously used keys. The cryptographic period may be fixed (one day, one week, etc.) or may vary, depending on specified events (users resigning, possible compromises). When the same key is used for both the enciphering and deciphering operations, it is called a secret key algorithm. If different keys are used for the two operations and one is kept secret but the other is made public, then it is called a public key algorithm [7],[15]. (See article by Hellman, this issue.) The former case allows the same key to be used for both encryption and decryption by both users of a communication link. The latter case requires each user to have two keys; the public key of the other person and his own secret key. Each person must therefore know and protect a secret key even in a public key system.

\section{CRYPTOGRAPHIC SYSTEMS}

While a cryptographic algorithm specifies the mathematics or logic of the encryption and decryption transformations, the cryptographic system specifies how the

\section{The security provided by a cryptographic system depends greatly on how the cryptographic algorithm is implemented and used in a particular application over and above the protection provided by the algorithm itself.}

algorithm shall be implemented and used in a particular application. While there are numerous cryptographic algorithms which may be developed, there are even more numerous ways that they may be applied in any particu- 
lar environment. The security provided by a cryptographic system depends greatly on the system itself over and above the cryptographic protection provided by the algorithm. The security can be either greater than or less than that fundamental to the algorithm.

A cryptographic system can either be added onto a communication system or integrated into it. Historically, the communication system already existed and the cryptographic system was added onto it in what was hoped to be a transparent manner. Stand-alone units containing a cryptographic device (a device implementing the cryptographic algorithm) would be placed between the communication device (modem) and the terminal (keyboard, printer, telephone). Such units provided electrical power, physical security, interface logic, and key entry facilities. They were designed to operate in pairs on each end of a communication circuit. Ciphertext and cryptographic control information was passed between them. Plaintext appeared outside the secure communication circuit. Cryptographic keys were manually generated and securely distributed to the units where they were manually entered at the beginning of a fixed cryptographic period. Great care was taken to assure that only ciphertext was ever transmitted down the circuit and that the key could not be obtained from the unit in any manner. Ciphertext was of ten transmitted at a constant rate, even though no data was entering the unit for transmission. Switching of the ciphertext from one circuit to another was not possible.

Cryptographic systems can be integrated into a communication system at various levels in the protocol hierarchy. Fig. 1 shows the typical diagram used when discussing communication protocols. An integrated. cryptographic system can provide more security and ser- vices than an add-on system. The services depend on the level of integration. The cryptographic system can be controlled by the user or the application system, or both. A user-controlled cryptographic system provides sufficient tools for a user to generate, distribute, and store keys, enable and disable the cryptographic functions, and maintain data that can be accessed only by that user. A system-controlled cryptographic system automatically provides these services for a user with little or no knowledge of such activity by the user. Security can be provided at the user level (user-to-user), the terminal level (terminal-to-terminal or computer), or the system level (system-maintained data).

\section{THE FEDERAL DATA ENCRYPTION STANDARD}

A Data Encryption Standard (DES) was published by the National Bureau of Standards [11] which specifies a cryptographic algorithm for use by the Federal Government in certain information processing applications. The standard is a mathematical description of an encryption algorithm and its inverse decryption algorithm. Both are based on the use of a secret key which must be the same for the encryption and decryption operations. References [2]-[4], [13], and [14] contain detailed descriptions of the standard, the fundamental level of security provided by the algorithm, and general descriptions of its applications.

The algorithm is designed to encipher and decipher blocks of data consisting of 64 bits under control of a 64-bit key. For any possible key [ 56 bits of the key are used by the algorithms and 8 bits are used for error detection during key management), all possible combinations of 64-bit inputs result in all possible combina-

LOCAL

HIERARCHY OF INTERFACE LEVELS

TERMINAL

USER PROTOCOL (LEVEL 4)

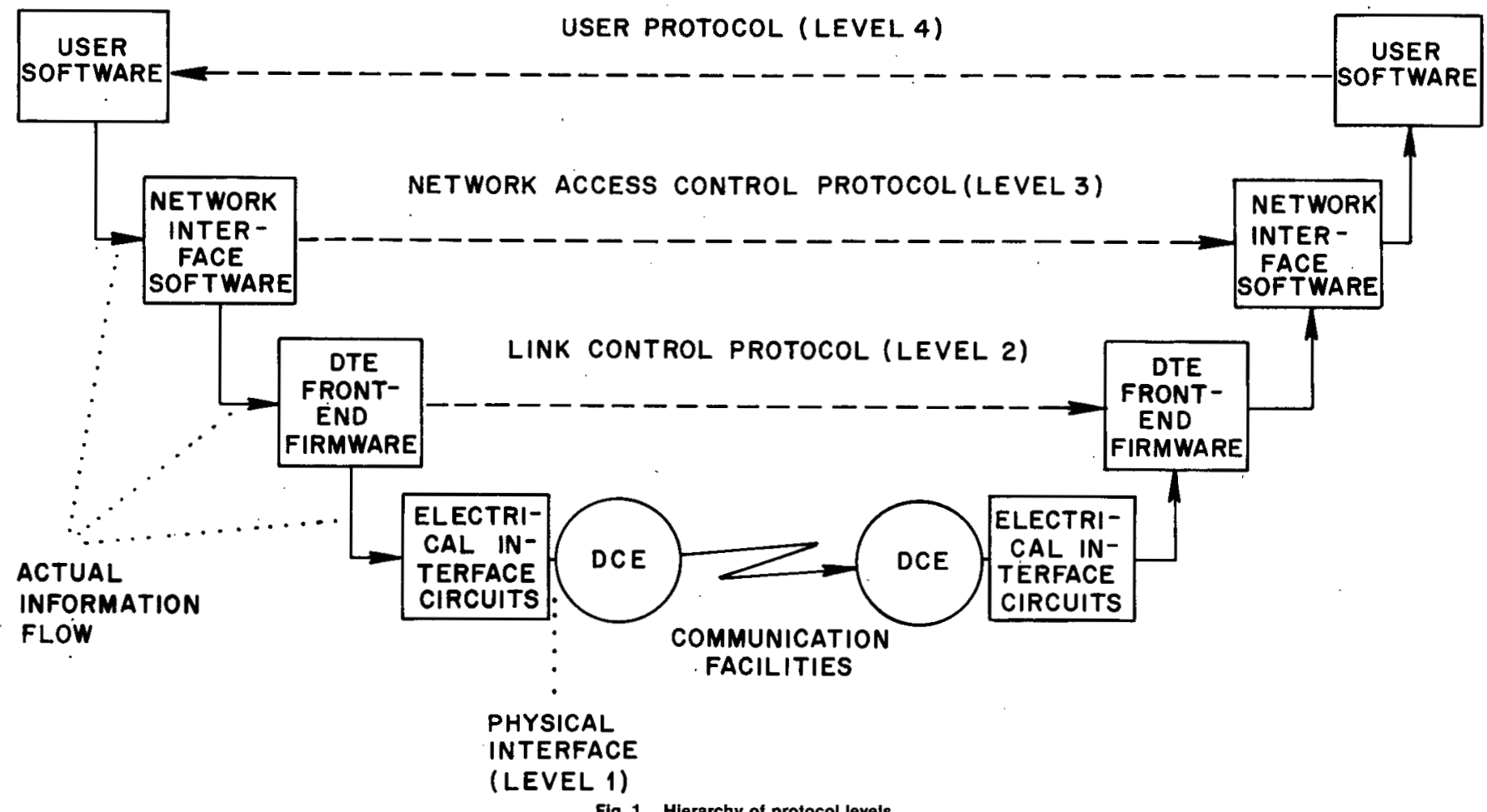

Fig. 1. Hierarchy of protocol leveis. 
tions of outputs. The decryption operation is the inverse transformation. For a particular key, the same, input results in the same output. Since the result of either operation depends on every bit of input and every bit of the 56-bit effective key, any change causes every bit of the result to change with equal probability. Thus, a mistake in entering the key or the ciphertext or in the decipherment process results in a radically different output. This characteristic can be used to detect intentional or accidental errors in a communication system.

The DES algorithm has been implemented in integrated 'circuits which have been validated by NBS as complying with the standard [12]. To date, five devices have been validated. All produce identical results given the same data input and key. Each manufacturer, however, has provided various control functions for the devices, optimized various features of the devices (speed, cost, size, power consumption), and built them to fill an anticipated need in the marketplace. For communications purposes, additional standards are required to assure that these devices are able to communicate with other devices. Similar to the U.S. telephone system in which all (existing) telephones can communicate with one a nother, it is desirable that all computer terminals and peripheral devices be able to communicate with one another. However, similar to the early telephone system (At one time telephones in different areas, even though only across the street, could not communicate), many existing computer terminals and peripheral devices cannot interoperate. Communication standards and protocols have minimized the number of codes, speeds, physical interfaces, and protocols somewhat, but many devices still cannot communicate on a network without significant modifications or enhancements. Federal standards [11] and American National Standards Institute standards are being developed for the application of the Data Encryption Standard in data communications.

\section{AN EXAMPLE OF NETWORK SECURITY}

The National Bureau of Standards is developing a local data network intended to serve up to a thousand users of equipment having dissimilar device characteristics [5]. The objectives of the network include providing fully connected communication capability among terminals, microcomputers, minicomputers, large host computers, and laboratory instruments; providing flow control of data among communicating devices; providing service among some 20 buildings at NBS using a single coaxial cable; and providing selective cryptographic protection to the network. The remaining portion of this paper describes the security provisions planned for this network.

A user views the network as a long cable with a number of hoxes connected into it. The boxes are microprocessor-based nodes called Terminal Interface Equipments, or TIE's. A TIE connects a network device (terminal, computer, or lab instrument) to the cable. Data travels through the cable at a signaling rate of one million bits per second. Data travels in logical entities called packets which appear (nearly) simultaneously at all TIE's. The function of the network is to transport data among TIE's. The purpose of network security is to assure that only the intended TIE can "read" the data, even though the

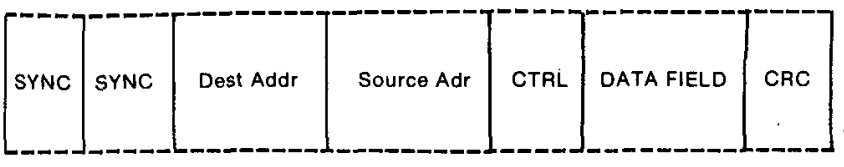

The SYNC characters are used for synchronization. The destination address (two bytes) is followed by the source address (two bytes). CTRL is a control byte for the network. The DATA FIELD may contain 0-128 data bytes. The CRC is a 16-bit error detection field for the packet.

Fig. 2 Data packet in the NBS network

normal protection features of the TIE have been circumvented and the data has been copied.

Every data packet on the network has the same form (see Fig. 2). Each contains a destination address, a source address, a data field, an error detection field, and control information. The data field contains $0-128$ bytes of information. Each address field contains 16 bits.

Each user of the network has a TIE connecting a device to the network. Each TIE can establish a single virtual connection to any other TIE to which data may be passed. A packet is assembled in the memory of a TIE before it is transmitted. Every TIE monitors the cable for a NOT BUSY condition. A TIE that has a packet ready for transmission begins sending when the NOT BUSY condition exists. Procedures are automatically invoked for retransmitting a message if more than one TIE attempts to initiate transmission concurrently. Every TIE monitors all transmissions, looking for packets having its destination address and originated by the TIE with which it has established a virtual connection.

The electronic equipment of a secure TIE consists of the network circuit board and the user circuit board of an ordinary TIE plus a security circuit board. The TIE is connected to the cable via the network board, which implements the protocols for network communication and collision avoidance. The user board contains a microprocessor and sufficient memory to hold the program which controls the TIE. The security board contains a DES device and a read-only memory chip containing the cryptographic key of the TIE as well as read-write memory for storing user keys and session keys. Data that is to be encrypted is sent to the security board from the user board. The presence, or absence, of the security board can be sensed by the user board. All TIEs have physical space for a security board, but only contain one if secure communication is desired for the associated device. Thus users not requiring secure communication pay no penalty for security.

\section{CRYPTOGRAPHIC PROTOCOL}

Cryptography is being integrated into the NBS network as a user/system controlled feature. Only the data field of any packet will be encrypted. A connection between any two devices can be established in either a regular or a secure mode. A secure connection requires that both TIE's in the connection contain security boards. A regular connection request has the following sample format:

\section{CON:1723.}

Assuming that the user is issuing the request from TIE 1711, a connection is established between TIE 1711 and TIE 1723. 


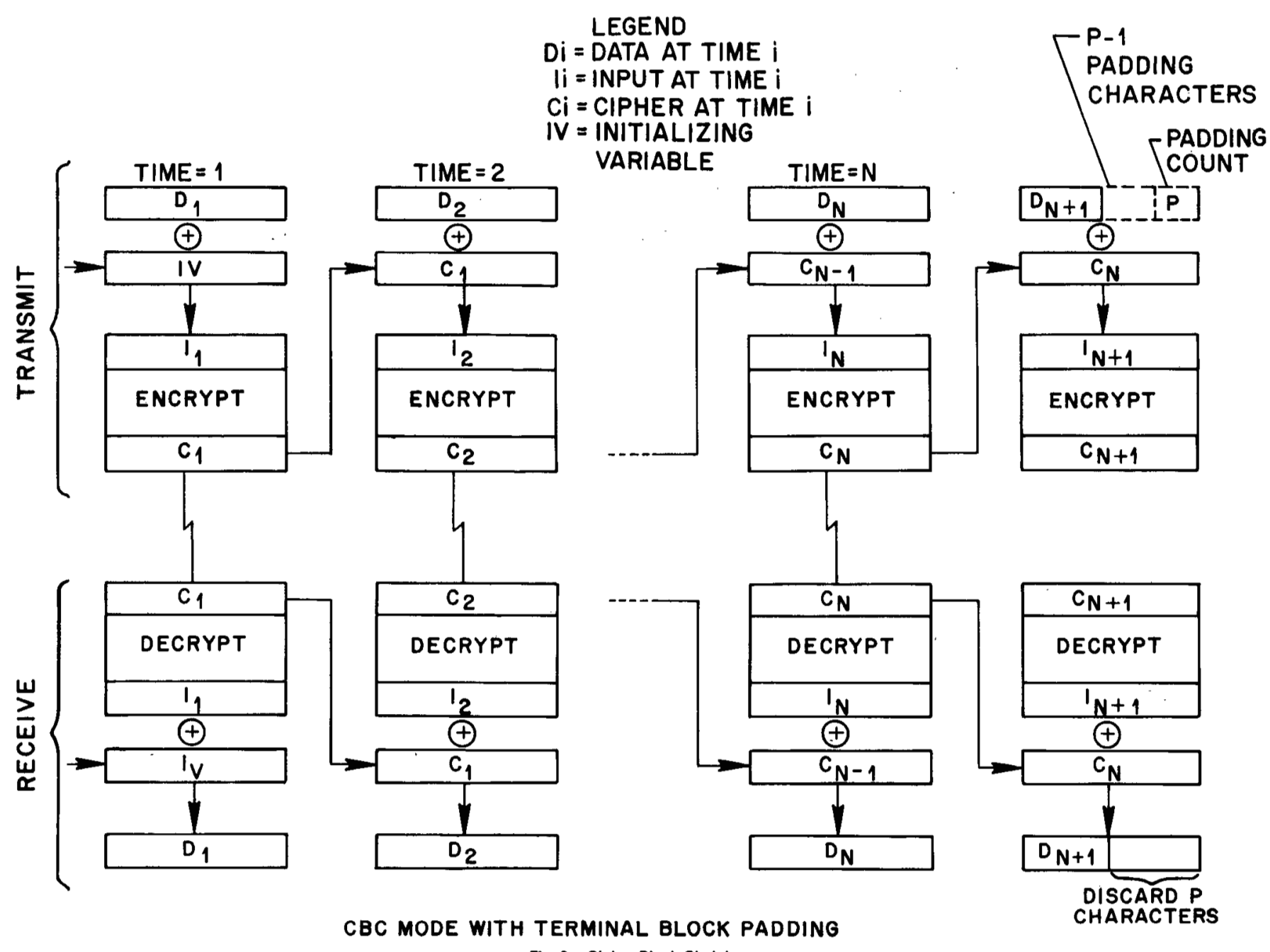

Fig. 3. Cipher Block Chaining

Assuming that a terminal is identified by its TIE and that a terminal also means a host computer, then a secure connection is similarly established in one of three ways: terminal-terminal, user-terminal, user-user. A mail system is also possible for secure messages for a particular person who is not present at the terminal. The message is stored in ciphertext form to be retrieved at a later time when the user is available.

Secure data will be encrypted with the DES using the Cipher Block Chaining [11],[16] mode of operation (Fig. 3). The data field of each packet will be encrypted as a separate entity. This formatted plaintext packet (Fig. 4) will be encrypted in its entirety before being transmitted. Anyone monitoring network traffic could receive the encrypted packet, but only the TIE with the correct key can decrypt the packet. The communication

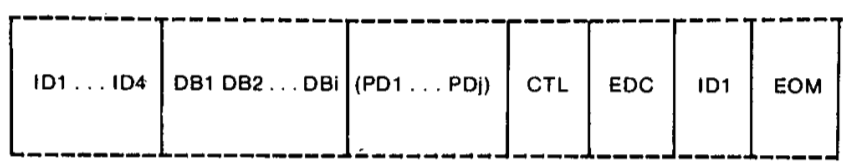

ID1 through ID4 is an extendable packet identification number $(0-268,435,455)$. DB1 through $\mathrm{DBi}$ are the data bytes to be transmitted. PD1 through $\mathrm{PDj}$ are 0.7 padding bytes used to assure that a multiple of 8 bytes are contained in the encrypted packet. $C T L$ is an encryption control field. EDC is an encryption error detection code. ID1 is a repeat of the first byte of the packet identification number and EOM is an end-of-packet flag.

Fig. 4 Data fleld of an encrypted packet security problem is thus reduced to a key management problem.

Once a secure connection is established, it remains secure until the connection is broken. The same key is used for both directions in the connection. As soon as the connection is broken, the key is destroyed.

\section{KEY MANAGEMENT}

Key management is a critical aspect as a cryptographic system. Physical security and operational reliability are related aspects. Key management for the NBS secure network will be performed by two identical microprocessor systems dedicated to the task of key management. These two systems are called Network Security Centers or NSC's. The systems will be physically isolated and individually protected. They will appear to the network as two TIE's with a "rotary" address sequence (if the first TIE is busy, the second will automatically be tried). The hardware, software, and data bases of the two NSC's will be identical.

The key management scheme for the NBS network will be based on a hierarchically structured set of cryptographic keys [10]. The hierarchy will include master keys, submaster keys, and session keys. Master keys will only be stored in physically secured memory within or close to the DES devices. Submaster keys will be enciphered under the master keys and will themselves be 
used to encipher other keys. Session keys will be generated by the NSC's and distributed to the security boards in the TIE's participating in a secure communication. Session keys will have a lifetime equal to the length of the session. Master keys will have a fixed, finite lifetime unless they have been compromised. Submaster keys will have a lifetime selected by a user.

The objective of the key management scheme is to establish a secure connection between TIE's in such a manner that the TIE's (hence the connected terminals) are authenticated, the users are authenticated (if requested by the initiating user) and a one-time session key is distributed to the TIE security boards for data protection. Several design approaches are currently under consideration. The operational implementation will depend on factors such as network security management, methods of manually protecting master keys, and overhead in establishing a secure connection.

\section{SECURE CONNECTIONS}

The key management system presently under consideration uses one key in each TIE for authentication of the TIE (and its attached terminal/computer) and one key for each user for authentication. All terminal and user keys are also stored in each NSC. A master key in each NSC is used to encrypt lists of all terminal keys and user keys. A user desiring a secure terminal to terminal connection issues the following exemplary command:

\section{TTS: 1723 .}

TTS stands for terminal-terminal secure and 1723 is the identifier of the terminal (TIE) that is being "called." The "calling" TIE contains a program to establish a secure connection to the "called" TIE in the following manner. Assume that the user is making the request from TIE 1711. A regular connection is made by TIE 1711 to the TIE of the NSC. The following message is transmitted to the NSC by TIE 1711:

$$
\text { TTS:1711:1723. }
$$

The NSC, upon receiving this message, acts as a service center in the establishment of a secure connection between terminal 1711 and terminal 1723. The NSC finds the terminal keys (encrypted under the NSC master key) for terminals 1711 and 1723 in its terminal key storage. Then using the DES it generates a random number which is defined to be the session key encrypted under the NSC master key. The NSC contains an integral DES device and does not require a security board in its TIE. The NSC decrypts TK1711 (the key for terminal 1711), TK1723, and the encrypted session key. After setting the parity bits of the session key, the NSC encrypts the session key (SK) using TK1711 and again using TK1723. It sends to terminal 1711 the following formatted message:

TTS:1711:1723:E-TK1711(SK):E-TK1723(SK).

E-TK1711(SK) means that the session key (SK) is encrypted (E) under the terminal key of 1711 (TK1711). TIE 1711 thus receives the session key encrypted under its own key as well as the key for TIE 1723 .

TIE 1711 then generates a random number called the initial vector (IV). The IV is used to randomize the first block of each packet transmitted during a secure connec- tion. After a regular connection is made to TIE 1723 , TIE 1711 transmits the following:

$$
\text { TTS:1711:1723:E-TK1723(SK):E-SK(IV). }
$$

TIE 1723 uses its stored terminal key to decrypt the SK. To verify that the SK received by TIE 1723 is the same as the SK received by TIE 1711 , the IV is decrypted using SK1723 (i.e., the SK received by TIE 1723), the first 32 bits of the 64-bit IV are complemented, and the modified IV is encrypted under SK1723. TIE 1723 transmits the following formatted message to TIE 1711:

$$
\text { TTS:1723:1711:E-SK1723(modified IV). }
$$

TIE 1711 then decrypts the modified IV with SK1711 and compares it with the IV originally sent. The first 32 bits should be complemented and the last 32 bits should be identical. If correct, the following message is transmitted:

$$
\text { T'TS:1711:1723:OK. }
$$

If incorrect, the following message is transmitted:

$$
\text { TTS:1711:1723:BAD. }
$$

If the connection is $O K$, all transmissions between the TIE's are encrypted in Cipher Block Chaining (CBC) mode using the SK and the IV. If the connection is BAD, the connection is aborted.

\section{USER-USER CONNECTION}

The previous section described a terminal-terminal connection in which the identity of the user on either end of the connection was unimportant. In many private communications, the identity of the calling or called party is important. The following describes the procedures planned for the secure NBS network in user-user connections. Terminal-user or user-terminal connections will not be described in this paper; they simply use the appropriate procedures established for each end of the secure connection.

A user (having identity U100) seeking to establish a private connection to another user (having identity U200) must supply the following formatted message to the TIE:

\section{UUS:1723:U100:U200:UK100,}

This requests that the "calling" TIE (assume TIE 1711) establishes a secure connection to U200 at terminal 1723 and supplies to TIE 1711 the user key for U100. It is assumed that U200 is present at terminal 1723 .

TIE 1711 transmits the following formatted message to the NSC:

\section{UUS:1711:1723:U100:U200.}

The NSC finds the terminal keys for 1711 and 1723 in the encrypted terminal key storage and the user keys for U100 and U200 in the encrypted user key storage. The NSC generates a random session key encrypted under NSC master key. The five encrypted keys are used to produce the following formatted message which is sent to TIE 1711:

$$
\begin{gathered}
\text { UUS:1711:1723:E-UK100(E-TK1711(SK)): } \\
\text { E-UK200(E-TK1723(SK)). }
\end{gathered}
$$


TIE 1711 then obtains the session key SK1711 by decrypting the received encrypted key using its TK1711 and UK100 contained in its key storage.

TIE 1711 produces the following message and, after establishing a connection to TIE 1723 , transmits the following message:

\section{UUS:1711:1723:U100:U200:E-UK200(E-TK1723(SK)): E-SK1711(IV).}

Upon receiving this message (in plaintext), TIE 1723 requests that U200 supply user key (U200) by printing the following message on the terminal associated with the TIE:

\section{TIE: Connection Request from U100; Please supply U200 Key:}

Upon entry of the U200 key, TIE 1723 deciphers the session keys using TK1723 and UK200, deciphers the IV using the deciphered session key, modifies the IV as previously described, enciphers the modified IV, and transmits the following formatted message to TIE 1711:

UUS:1723:1711:U200:U100:E-SK1723(Modified IV).

TIE 1711 deciphers the modified IV with SK1711, compares it with the original IV, and the secure connection is considered established if the IV has been properly modified. A message is sent (in plaintext) to TIE 1723, informing it if the connection is $\mathrm{OK}$ or BAD. If $\mathrm{OK}$, all further messages are enciphered in Cipher Block Chaining mode using the original IV.

Correctly receiving the modified IV at TIE 1711 verifies the identity of U100, U200, TIE 1711, TIE 1723 and verifies that the session key and the IV are correct and ready for use.

\section{DESIGN RATIONALE}

The initial design of the NBS secure network key management scheme is based on key management concepts $[1],[2],[9],[10]$ that have been reduced to the minimum number of connections and messages necessary to establish a secure connection. It must be recognized that tradeoffs are necessary between security and convenience in such a design. Convenience was emphasized in this example for easier understanding. Only five messages are required to establish and verify a secure connection. Because the only errors detected are key parity errors and improper key distribution errors, the only error reaction is to abort the attempted connection. As a result, the cause of failure to establish a connection cannot be easily surmised.in this simple design.

Implementation of these network security procedures (planned to be operational in 1980) will depend on additional reliability and security requirements identified during early network development. For example, additional anti-spoofing procedures will be provided to prevent a penetrator from recording an encrypted terminal-terminal connection procedure and subsequent communication and then replaying it back through the "calling" terminal after gaining physical access to the terminal (but not gaining access to the terminal key).

Requirements for physical security of the TIE's and the * NSC's are expected to change during implementation of the network. Economy is presently a major design cri- teria. Physical locks on the TIE units which prevent unauthorized access to the TIE key will be provided. TIE keys will be generated at the NSC, written onto a Read Only memory (ROM) device, and physically transported to a TIE. User keys will be generated at the NSC and issued to users, either in printed form, on a magnetic striped card, or in a ROM device to be inserted in a port of the TIE or some combination thereof. Administrative procedures will be established so that user keys can be changed by both the user and the system.

Physical security of the NSC will include protection of the master key in the DES device and protection of the random access memory while the computer is operating. Keys stored on the disks will be encrypted with the master key, and hence will not require physical protection. However, if a master key is compromised, all key lists in both NSC's must be rebuilt and the master key in both NSC's must be changed. Dual NSC's are used for reliability purposes.

\section{CONCLUSIONS}

Security of computer communications can be provided through cryptography. Cryptographic algorithms and devices implementing them are available for this pur-

\section{Through the use of small dedicated computers for storing and generating keys, the problems of key management can be reduced to manageable proportions.}

pose. Communications protocols are being established which makes the transparent use of cryptography practical. Through the use of small, dedicated computers with sufficient data storage and processing capability for storing and generating keys, the problems of key management can be reduced to manageable proportions. If public-key cryptographic algorithms [7],[15] are developed which can be effectively used for keymanagement functions, the physical security requirement for NSC's will be eliminated or reduced to that of a public key distribution system. By enhancing the capability of the NSC and increasing the number of messages that it can handle, additional security features can be provided such as auditing, security surveillance, and host computer security interfacing. Comments and suggestions in these areas as well as in means of extending the protocol for establishing communication security will be welcomed by the author.

\section{BIBLIOGRAPHY}

[1] D. Branstad, "Security aspects of computer networks," in AIAA Conf. Proc., Huntsville, AL, Apr. 1973.

[2] -, "Encryption protection in computer data communications," in 4th Data Commun. Symp. Proc., IEEE Computer Society, Oct. 1975.

[3] D. Branstad, J. Gait, and S. Katzke, "Report of the Workshop on Cryptography in Support of Computer Security,". NBSIR 77-1291, National Bureau of Standards, Sept. 1977.

[4] D. Branstad, "Computer security and the Data Encryption Standard," NBS Special Publ. 500-27, National Bureau of Standards, Feb. 1978. 
[5] R. Carpenter, J. Sokol, and R. Rosenthal, "A microprocessor-based local network node," in COMPCON Proc., IEEE Computer Society, Sept. 1978.

[6] Data Encryption Standard, Federal Information Processing Standard (FIPS) Publ. 46, National Bureau of Standards, U.S. Department of Commerce, Jan. 1977.

[7] W. Diffie, and M. Hellman, "New directions in cryptography," IEEE Trans. Inform. Theory, Nov. 1976.

[8] - - "Exhaustive cryptanalysis of the NBS Data Encryption Standard," IEEE Computer, June 1977.

[9] S. Ehrsam, S. Matyas, C. Meyer, and W. Tuchman, "A cryptographic key management scheme for implementing the Data Encryption Standard," IBM Syst. J. vol. 17, no. 2 , 1978.

[10] J. Everton, "A hierarchical basis for encryption key management in a computer communications network," in Trends and Applications: 1978 Proc., IEEE Computer Society, May 1978.

[11] Federal Standard 1026 (proposed), "Telecommunications: Compatibility requirements for use of the Data Encryption Standard," National Bureau of Standards, Oct. 1977.

[12] ]. Gait, "Validating the correctness of hardware implementations of the NBS Data Encryption Standard," NBS Special Publ. 500-20, National Bureau of Standards, Nov. 1977.

[13] P. Meissner, "Report of the workshop on estimation of Significant Advances in Computer Technology," NBSIR 76-1189, National Bureau of Standards, Dec. 1976.
[14] C. Meyer, and S. Matyas, "Cryptography: A new dimension in computer data security," KETRON, Inc., 1978.

[15] R. Rivest, A. Shamir, and L. Adleman, "On digital signa'tures and public-key cryptosystems," Tech. Memo 82, MIT Lab. for Computer Science, Apr. 1977.

[16] L. Tuckerman, "Block-cipher cryptographic system with chaining," U.S. Patent 4078152 assigned to the IBM Corporation, Mar. 1978 .

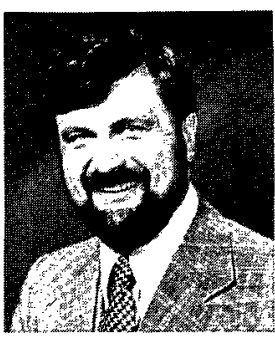

Dennis Branstad is the project leader for computer security at the Institute for Computer Sciences and Technology within the National Bureau of Standards. Since 1973, he has been responsible for the development of the Federal Data Encryption Standard. He has written several papers on the use of cryptography in computer network security applications. He has chaired or participated in several Federal computer security standards committees and in several ANSI activities developing cryptographic standards in financial transactions and data communications. He holds a Ph.D. degree in Computer Science from Iowa State University and is an Adjunct Professor of Computer Science at the University of Maryland.

\section{Communications Privacy}

\section{at NTC'78}

Included in the NTC'78 program at Birmingham is a session on Communications Privacy organized by Michael Willett of the University of Virginia. R. E. Lennon and S. M. Matyas of IBM will present a new key distribution method in which the data-encrypting key is a composite of random data generated at each node. Robert M. Fenichel of National Communications Systems will review federal and national activity in the data encryption standards area. He will also review the methods for using the NBS encryption algorithm in data communications. John Michael Williams of System Development Corporation will review the DES controversy and discuss the use of public key techniques in the context of system issues still unresolved. Leonard Adleman and Ronald Rivest of M.I.T. will describe the use of public key cryptography in communications system design. The text of their paper appears in this issue. Finally, Michael Willett will survey the latest recommendations in the area of privacy and government legislation, with emphasis on those issues which still have an impact on the telecommunications industry.

\section{VOICE SECURITY - anywhere PaIVACOn Suitcase Scrambler}

Communicate in utmost privacy on any standard telephone, wherever you go. The speech scrambler's acoustical telephone coupler permits wire free operation. Over 6 million code selections. Codes change twice per second to deter decoding. Impact resistant suitcase with tamper-proof lock.

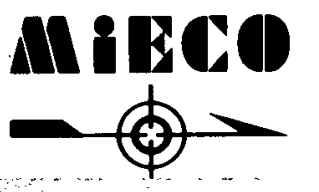

Division: Polarad Electronics Corp. 109 Beaver Court, Cockeysville, Md. U.S.A. 21030 - Tel. (301) 667.4660 TWX 710-862-9068 MIECO COYE

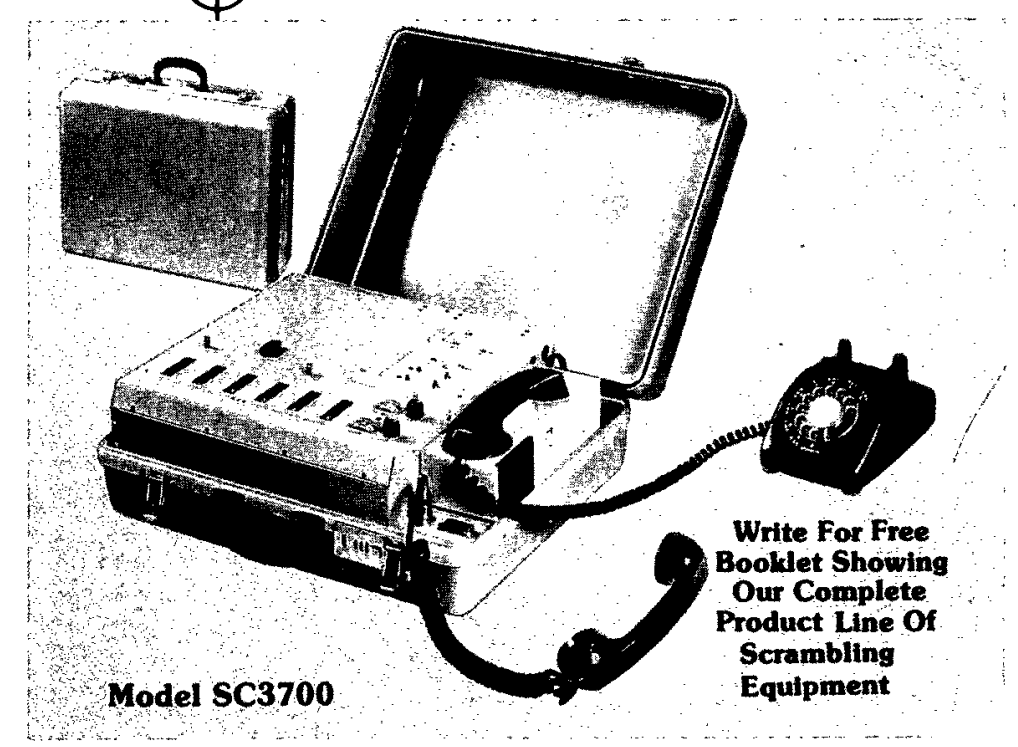

